
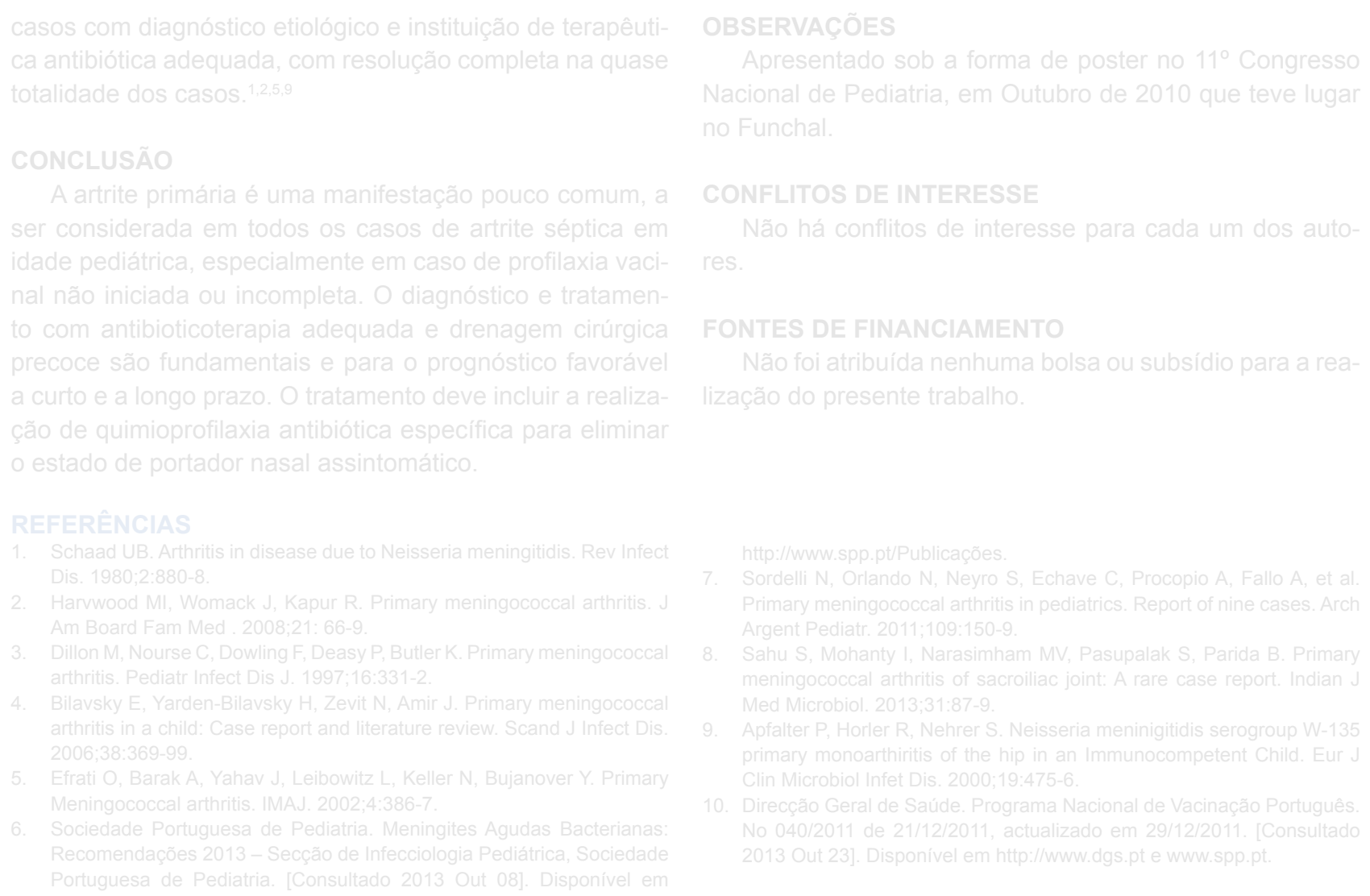

\title{
Nefrocalcinose e Necrose Gorda do Tecido Celular Subcutâneo
}

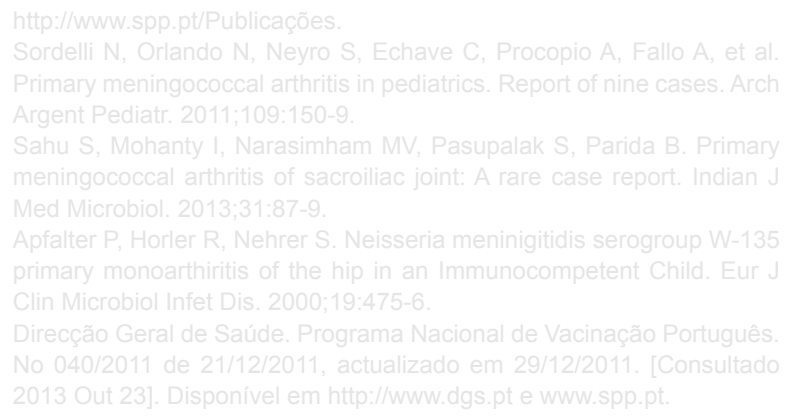


Keywords: Hypercalcemia; Fat Necrosis; Infant, Newborn; Nephrocalcinosis; Subcutaneous Fat.

\section{INTRODUÇÃO}

A necrose gorda do tecido celular subcutâneo (NGS) do recém-nascido (RN) é uma paniculite pouco frequente, transitória e auto-limitada, que surge até quatro semanas após o nascimento..$^{1-4}$ Apesar da etiopatogenia desta entidade não estar bem esclarecida, estão identificados vários fatores de risco para a sua ocorrência, nomeadamente complicações da gravidez (diabetes gestacional, pré-eclâmpsia), complicações do parto (macrossomia, trauma, asfixia) e intervenções terapêuticas (hipotermia terapêutica, hipotermia para cirurgia cardíaca). ${ }^{1,3-8} \mathrm{~A}$ evolução é habitualmente favorável, mas podem surgir complicações potencialmente graves, nomeadamente hipercalcémia, que pode surgir tardiamente na história natural da doença, até seis meses após aparecimento das lesões cutâneas. ${ }^{9-11}$ Os doentes com hipercalcémia persistente podem desenvolver calcificação metastática em diferentes órgãos. ${ }^{8} \mathrm{~A}$ nefrocalcinose pode surgir como complicação ${ }^{5}$ e pode condicionar evolução para doença renal crónica (DRC). Descreve-se a abordagem de um caso de NGS complicada por hipercalcémia e nefrocalcinose.

\section{CASO CLÍNICO}

Recém-nascido do sexo feminino, com antecedentes pessoais de gestação de termo, sem intercorrências, parto por cesariana por sinais de sofrimento fetal, com Índice de APGAR ao $1^{\circ}, 5^{\circ}$ e $10^{\circ}$ minuto de $3 / 6 / 6$ respetivamente e peso ao nascer de $4995 \mathrm{~g}$. Transferida nas primeiras horas de vida para um hospital central e universitário, tendo sido submetida a hipotermia passiva durante o transporte, com reaquecimento à chegada (tempo total de hipotermia seis horas). Ao sétimo dia de vida (D7) surgiram lesões cutâneas nódulo-papulares, eritematosas, dispersas no dorso, região glútea e membros superiores. O quadro clínico era compatível com diagnóstico de NGS. Os valores séricos de cálcio $(\mathrm{Ca}(\mathrm{s}))$ permaneceram normais e a evolução clínica

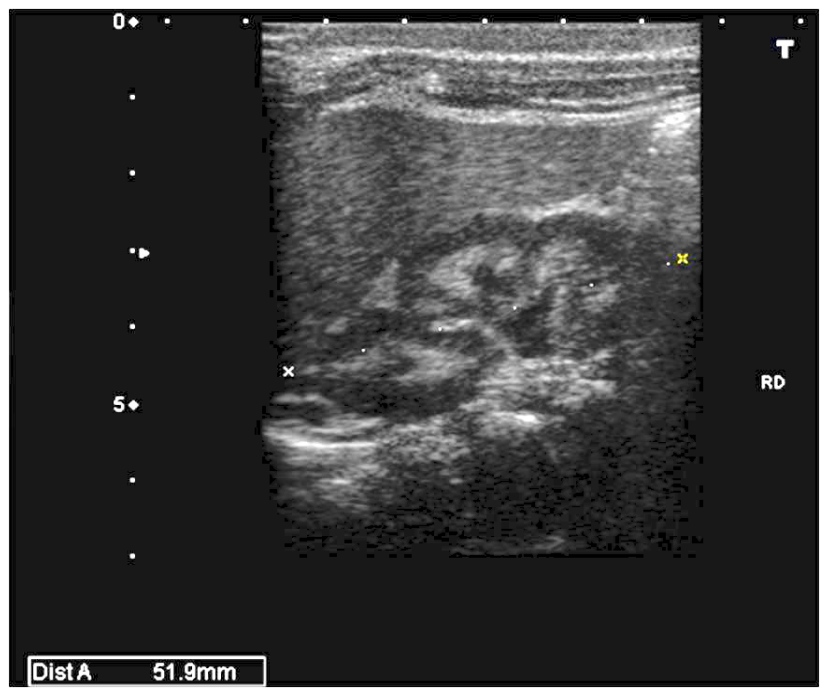

Figura 1 - Rim direito com hiperecogenicidade à periferia das pirâmides renais traduzindo alterações de nefrocalcinose medular foi favorável. Teve alta em D20, com os diagnósticos de encefalopatia hipóxico-isquémica ligeira, hipertensão pulmonar persistente, miocardiopatia hipertrófica, sépsis precoce e NGS; sob aleitamento artificial com leite adaptado formula 1 , sem suplementação com vitamina $D$. Reinternada em D37 por hipotonia axial, dificuldade alimentar, reflexo de sucção fraco e reaparecimento de novas lesões de NGS acompanhadas de dor intensa à mobilização. A avaliação analítica revelou hipercalcémia grave (Ca(s): $16,2 \mathrm{mg} / \mathrm{dL}$ (Vr: 8,6-10,0); cálcio sérico corrigido para a albumina sérica: $17,2 \mathrm{mg} / \mathrm{dL}$ (Vr: 8,6-10,0); cálcio ionizado: $2,15 \mathrm{mmol} / \mathrm{L}$ (Vr: 1,13-1,32); cálcio urinário $(\mathrm{Ca}(\mathrm{u}))$ : $14 \mathrm{mg} / \mathrm{dL}(\mathrm{Vr}: 6,7-$ 21,3); creatinina urinária $(\mathrm{Cr}(\mathrm{u})): 4,3 \mathrm{mg} / \mathrm{dL}(\mathrm{Vr}: 28-259)$; relação cálcio/creatinina urinária $3,3(\mathrm{~N}<0,8)$; fósforo sérico $(\mathrm{P}(\mathrm{s})): 4,8 \mathrm{mg} / \mathrm{dL}(\mathrm{Vr}: 2,5-6,0)$ e PTH $28,2 \mathrm{pg} / \mathrm{ml}(\mathrm{Vr}: 14-72))$. Não foi feito o doseamento da $1,25(\mathrm{OH})_{2}$ Vitamina D. Os exames imagiológicos efetuados para pesquisa de calcificações metastáticas incluíram uma ressonância magnética crânio-encefálica e ecocardiograma que não evidenciaram a presença de calcificações. A ecografia renal e vesical revelou "(...) presença de sinais de nefrocalcinose medular bilateral e litíase no seio renal esquerdo com cálculo medindo cerca de 4,5 mm (...)" (Fig.s 1,2). A terapêutica instituída para controlo da hipercalcémia incluiu hidratação endovenosa, leite especial com aporte de cálcio de acordo com a dose mínima diária recomendada em função da idade, diurético expoliador de cálcio (furosemida $0,9 \mathrm{mg} / \mathrm{Kg} / \mathrm{dia}$ ) e prednisolona (1 $\mathrm{mg} / \mathrm{Kg} / \mathrm{dia})$. A corticoterapia foi mantida durante 20 dias e a evolução foi favorável. Verificou-se uma normalização do valor de $\mathrm{Ca}(\mathrm{s})$ total $(10,8 \mathrm{mg} / \mathrm{dL})$ e da relação cálcio/creatinina urinária $(0,5 \mathrm{mg} / \mathrm{mg})$. Aos dois anos de idade, apresenta-se com lesões cutâneas fibróticas cicatriciais, dispersas no dorso e membros (Fig. 3). Analiticamente mantém função renal normal (DFG: $86,5 \mathrm{ml} / \mathrm{min} / 1,73 \mathrm{~m}^{2}$ -Fórmula de Schwartz), $\mathrm{Ca}(\mathrm{s}): 10 \mathrm{mg} / \mathrm{dL}, \mathrm{P}(\mathrm{s}): 4,9 \mathrm{mg} / \mathrm{dL}$,

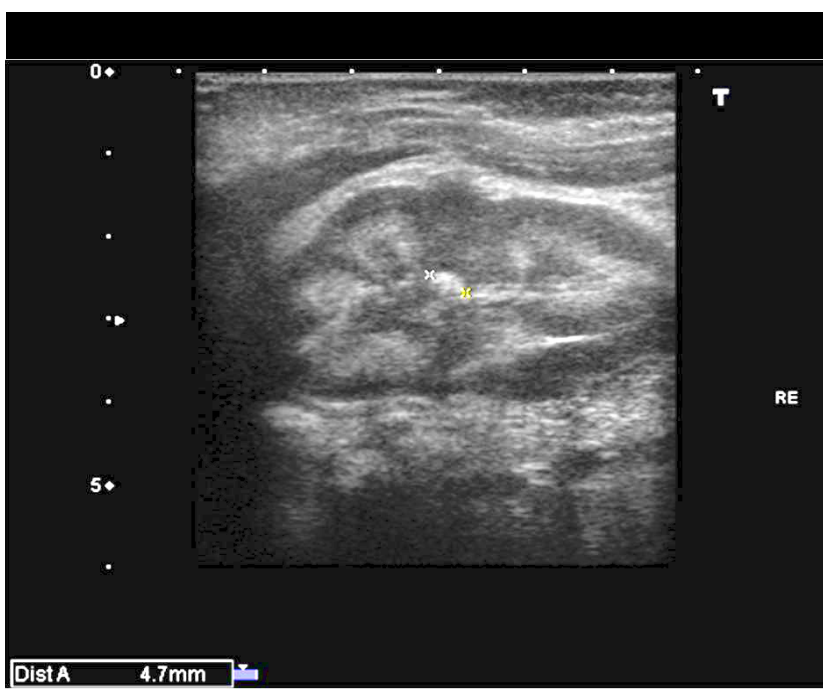

Figura 2 - Rim esquerdo com hiperecogenicidade à periferia das pirâmides renais traduzindo alterações de nefrocalcinose medular e litíase no seio renal esquerdo com cerca de $4,5 \mathrm{~mm}$ 


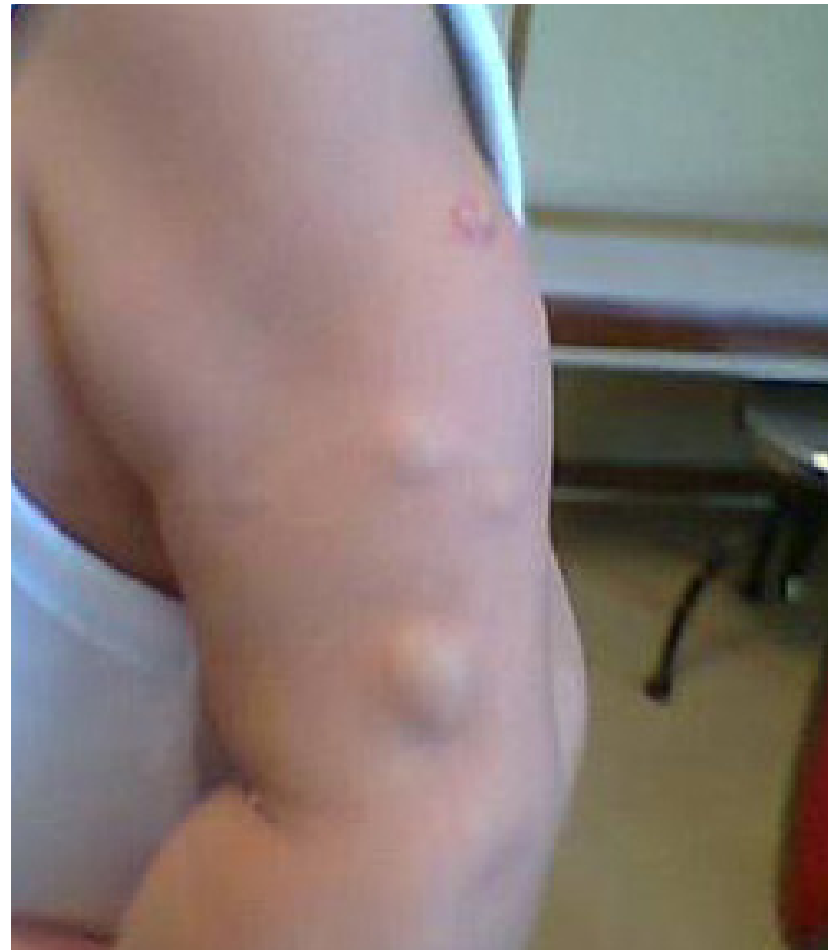

Figura 3 - Lesões cutâneas fibróticas cicatriciais secundárias a necrose gorda subcutânea

relação cálcio/creatinina urina: $0,39(\mathrm{~N}<0,5)$ e PTH: 22,0 $\mathrm{pg} / \mathrm{mL}$. A ecografia renal mantém sinais de nefrocalcinose medular sobreponível, sem sinais de litíase. Mantém acompanhamento multidisciplinar.

\section{DISCUSSÃO}

A NGS foi descrita pela primeira vez em 1926 por Harrison e McNee. ${ }^{5}$ Surge habitualmente em recém-nascidos de termo, apesar de poder afetar recém-nascidos pré-termo. ${ }^{1,6,11}$

O diagnóstico é clínico e caracteriza-se pelo aparecimento de nódulos e/ou placas subcutâneos eritemato-violáceos, de consistência dura, dolorosos, com localização predominante nas proeminências ósseas do dorso, braços, coxas, região glútea e face. ${ }^{1,4,6} \mathrm{O}$ exame anatomopatológico das lesões, por biópsia ou citologia aspirativa, ${ }^{1,3}$ permite a confirmação do diagnóstico e é caracterizado por necrose dos adipócitos, com cristais eosinofílicos em disposição radial, e infiltrado inflamatório composto por granulomas de histiócitos, células gigantes multinucleadas, neutrófilos e alguns linfócitos. O principal diagnóstico diferencial a ser estabelecido é com o esclerema neonatal. ${ }^{1,6}$

Neste caso o diagnóstico foi clínico, considerando as características e localização das lesões. Habitualmente a NGS tem resolução espontânea, mas podem surgir complicações como trombocitopénia, hipoglicémia e hipertrigliceridémia, em regra transitórias. ${ }^{1} \mathrm{~A}$ hipercalcémia é a complicação que deverá exigir maior atenção pela sua gravidade, ocorrendo em $28-69 \%$ dos casos. ${ }^{3}$ Manifesta-se no lactente de forma inespecífica, nomeadamente por letargia, irritabilidade, hipotonia, vómitos, poliúria, polidipsia, desidratação, obstipação e má progressão estaturoponderal, ${ }^{1}$ pelo que requer elevado índice de suspeição. No caso clínico, os sintomas e sinais de hipercalcémia estavam presentes à data do reinternamento e a avaliação analítica confirmou esta complicação.

A teoria etiopatogénica da hipercalcémia mais aceite, propõe uma produção ectópica de $1,25(\mathrm{OH})_{2}$ vit.D3 pelo infiltrado inflamatório presente nas lesões granulomatosas da NGS (e outras doenças granulomatosas como sarcoidose ou tuberculose), responsável pela estimulação da absorção intestinal de cálcio. ${ }^{3,5,10}$ Está demonstrada uma expressão aumentada da enzima 1a-hidroxilase, responsável pela hidroxilação de $25(\mathrm{OH}) \mathrm{D} 3$ em $1,25(\mathrm{OH})_{2} \mathrm{D} 3$, no infiltrado inflamatório de doenças granulomatosas como a NGS, apesar desta ser expressa predominantemente no rim. $^{2}$

Aproximadamente $30 \%$ dos doentes com hipercalcémia persistente e não corrigida desenvolvem nefrocalcinose ${ }^{11}$ podendo ainda ocorrer calcificação metastática de outros órgãos ou sistemas. . $^{1,5,8,9}$

O controlo da hipercalcémia requer hidratação endovenosa, restrição do aporte de cálcio e de vitamina $\mathrm{D}$ e diuréticos da ansa (expoliadores de cálcio). A corticoterapia, a calcitonina e os bifosfonatos são alternativas nos casos graves e refractários. ${ }^{1,4,10}$

Os autores alertam para a importância da monitorização regular (quinzenal) ${ }^{3}$ dos valores de cálcio sérico e urinário, no mínimo até seis meses após a resolução das lesões inflamatórias cutâneas. A suplementação com vitamina $D$ deve ficar suspensa nos primeiros seis meses de vida e sempre que detetada hipercalcémia ou complicações associadas, pelo risco iatrogénico de agravamento da hipercalcémia.

Os casos de nefrocalcinose descritos na literatura apresentaram resolução nos primeiros 24 meses, ${ }^{5}$ no entanto há possibilidade de evolução para DRC.

\section{CONCLUSÃO}

A hipercalcémia é uma complicação da NGS, por vezes tardia. A monitorização regular dos valores de cálcio sérico e urinário é essencial, no mínimo até seis meses após a resolução das lesões cutâneas, porque o diagnóstico precoce e a terapêutica adequada constituem a única possibilidade de prevenir complicações mais graves. A hidratação, o controlo do aporte de cálcio e a interrupção da suplementação com vitamina $D$ podem ajudar a minimizar os efeitos da hipercalcémia. A terapêutica farmacológica é necessária em alguns casos.

\section{CONFLITOS DE INTERESSE}

Os autores declararam a inexistência de conflitos de interesse.

\section{FONTES DE FINANCIAMENTO}

Não foi atribuída nenhuma bolsa ou subsídio para a realização do presente trabalho. 
1. Tran JT, ShET

1. Tran JT, Sheth AP. Complications of subcutaneous fat necrosis of the newborn: a case report and review of the literature. Pediatr Dermatol. 2003;20:257-61.

2. Farooque A, Moss C, Zehnder D, Hewison M, Shaw NJ. Expression of 25-hydroxyvitamin D3-1alpha-hydroxylase in subcutaneous fat necrosis. Br J Dermatol. 2009;160:423-5.

3. Akın MA, Akın L, Sarıcı D, Yılmaz I, Balkanlı S, Kurtoğlu S. Follow up during early infancy of newborns diagnosed with subcutaneous fat necrosis. J Clin Res Pediatr Endocrinol. 2011;3:216-8.

4. Akcay A, Akar M, Oncel MY, Kızılelma A, Erdeve O, Oguz SS, et al. Hypercalcemia due to subcutaneous fat necrosis in a newborn after total body cooling. Pediatr Dermatol. 2013;30:120-3.

5. Gu LL, Daneman A, Binet A, Kooh SW. Nephrocalcinosis and nephrolithiasis due to subcutaneous fat necrosis with hypercalcemia in two full term asphyxiated neonates: sonographic findings. Pediatr Radiol. 1995;25:142-4.

6. Mitra S, Dove J, Somisetty SK. Subcutaneous fat necrosis in newborn
- an unusual case and review of literature. Eur J Pediatr. 2011;170:110710.

7. Strohm B, Hobson A, Brocklehurst P, Edwards AD, Azzopardi D, Toby UK. Cooling Register. Subcutaneous fat necrosis after moderate therapeutic hypothermia in neonates. Pediatrics. 2011;128.

8. Dudink J, Walther FJ, Beekman RP. Subcutaneous fat necrosis of the newborn: hypercalcaemia with hepatic and atrial myocardial calcification. Arch Dis Child Fetal Neonatal Ed. 2003;88.

9. Vijayakumar M, Prahlad N, Nammalwar BR, Shanmughasundharam R. Subcutaneous fat necrosis with hypercalcemia. Indian Pediatr. 2006;43:360-3.

10. Tseng MH, Chu SM, Cheng CJ, Lien R, Shih IS, Lin SH. An infant with multiple subcutaneous nodules, hypercalcemia, and nephrocalcinosis: questions and answers. Pediatr Nephrol. 2013;28:2283-7.

11. Burden AD, Krafchik BR. Subcutaneous fat necrosis of the newborn: a review of 11 cases. Pediatr Dermatol. 1999;16:384-7.
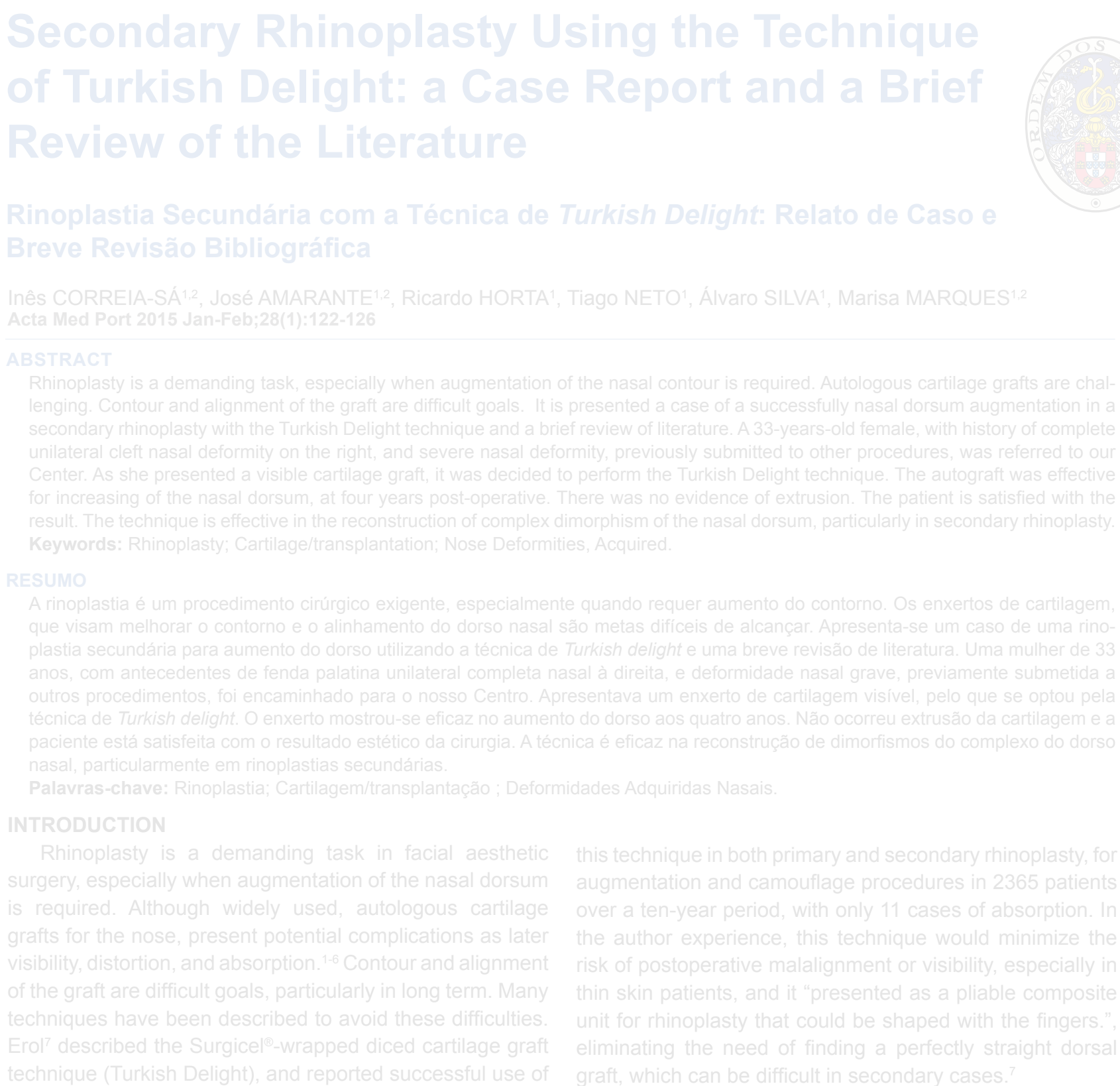


\section{Nefrocalcinose e Necrose Gorda do Tecido Celular Subcutâneo}

Acta Med Port 2015:28:119-122

Publicado pela Acta Médica Portuguesa, a Revista Científica da Ordem dos Médicos

Av. Almirante Gago Coutinho, 151

1749-084 Lisboa, Portugal.

Tel: +351218428 215

E-mail: submissao@actamedicaportuguesa.com

www.actamedicaportuguesa.com

ISSN:0870-399X | e-ISSN: 1646-0758

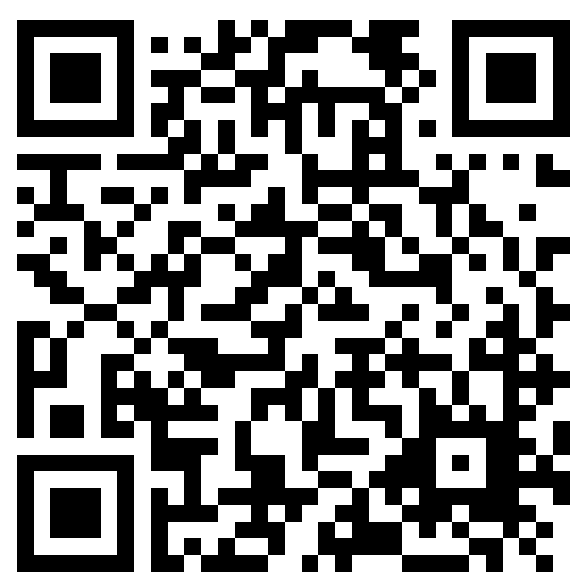

